University of Nebraska - Lincoln

DigitalCommons@University of Nebraska - Lincoln

\title{
$2-2012$
}

\section{Ferroelectric and multiferroic tunnel junctions}

Evgeny Y. Tsymbal

University of Nebraska-Lincoln, tsymbal@unl.edu

Alexei Gruverman

University of Nebraska-Lincoln, agruverman2@unl.edu

Vincent Garcia

CNRS/Thales, France, vncent.garcia@thalesgroup.com

Manuel Bibes

CNRS/Thales, France, manuel.bibes@thalesgroup.com

A. Barthélémy

CNRS/Thales, France

Follow this and additional works at: https://digitalcommons.unl.edu/physicstsymbal

Part of the Condensed Matter Physics Commons

Tsymbal, Evgeny Y.; Gruverman, Alexei; Garcia, Vincent; Bibes, Manuel; and Barthélémy, A., "Ferroelectric and multiferroic tunnel junctions" (2012). Evgeny Tsymbal Publications. 51.

https://digitalcommons.unl.edu/physicstsymbal/51

This Article is brought to you for free and open access by the Research Papers in Physics and Astronomy at DigitalCommons@University of Nebraska - Lincoln. It has been accepted for inclusion in Evgeny Tsymbal Publications by an authorized administrator of DigitalCommons@University of Nebraska - Lincoln. 

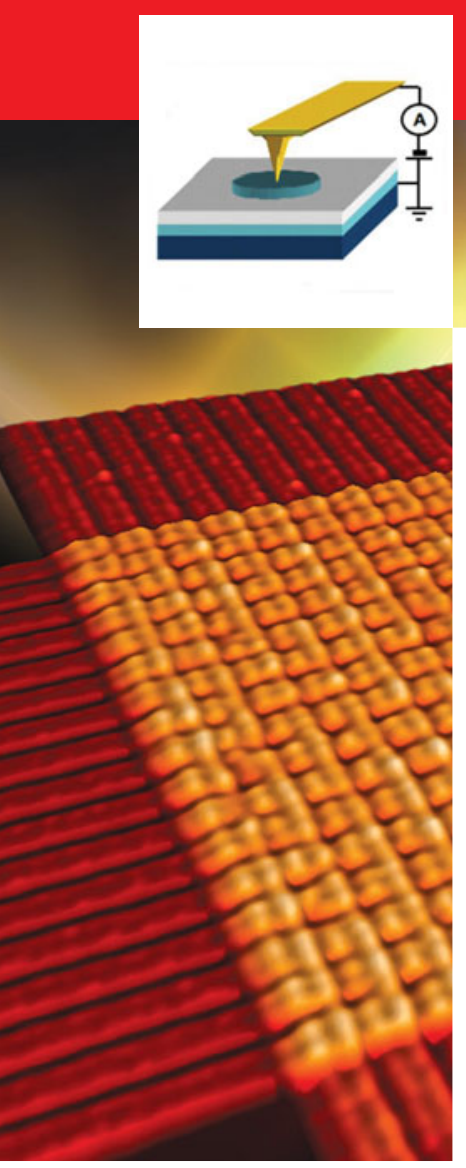

\title{
Ferroelectric and multiferroic tunnel
} junctions

\author{
E.Y. Tsymbal, A. Gruverman, V. Garcia, M. Bibes, \\ and A. Barthélémy
}

\begin{abstract}
The phenomenon of electron tunneling has been known since the advent of quantum mechanics, but continues to enrich our understanding of many fields of physics, as well as creating sub-fields on its own. Spin-dependent tunneling in magnetic tunnel junctions has aroused considerable interest and development. In parallel with this endeavor, recent advances in thin-film ferroelectrics have demonstrated the possibility of achieving stable and switchable ferroelectric polarization in nanometer-thick films. This discovery opened the possibility of using thin-film ferroelectrics as barriers in magnetic tunnel junctions, thus merging the fields of magnetism, ferroelectricity, and spin-polarized transport into an exciting and promising area of novel research. Nowadays, this research has become an important constituent of a broader effort in multiferroic materials and heterostructures that involves rich fundamental science and offers a potential for applications in novel multifunctional devices. The purpose of this article is to review recent developments in ferroelectric and multiferroic tunnel junctions. Starting from the concept of electron tunneling, we first discuss the key properties of magnetic tunnel junctions and then assess key functional characteristics of ferroelectric and multiferroic tunnel junctions. We discuss the recent demonstrations of giant resistive switching observed in ferroelectric tunnel junctions and the new concept of electrically controlling the spin polarization in magnetic tunnel junctions with a ferroelectric tunnel barrier.
\end{abstract}

\section{Magnetic tunnel junctions}

Electron tunneling is a quantum-mechanical effect, where electrons can traverse the potential barrier that exceeds their kinetic energy. This phenomenon has been known since the advent of quantum mechanics and reflects the wave nature of electrons. ${ }^{1}$ Electron tunneling can be realized in tunnel junctions that consist of two metal electrodes separated by a very thin insulating (e.g., $\mathrm{Al}_{2} \mathrm{O}_{3}$ or $\mathrm{MgO}$ ) or vacuum barrier. Numerous useful electronic devices are based on this phenomenon. For example, tunneling between two superconductors separated by a thin insulating layer, called a Josephson junction, has found important practical applications in superconducting quantum interference devices (SQUIDs), integrated circuits, and particle detectors. ${ }^{2}$ Electron tunneling lies at the heart of scanning tunneling microscopy (STM), which has become a conventional tool for studying the arrangement of individual atoms and molecules on surfaces. ${ }^{3}$ Field emission in the presence of a high electric field is another kind of electron tunneling, known also as Fowler-Nordheim tunneling, ${ }^{4}$ which is used as an electron source in flash memory, electron microscopy, and field emission displays.

Significant interest in electron tunneling has been triggered by the advent of spin-electronics (or spintronics), a technology aiming to harness the electron spin in data storage and processing, typically by utilizing heterostructures composed of magnetic and non-magnetic materials. ${ }^{5,6}$ Electron tunneling from a ferromagnetic metal electrode through a thin insulating barrier is spin-dependent. This is due to a disproportion in the number of electrons parallel and antiparallel to the magnetization of a ferromagnet, usually referred to as majority- and minority-spin electrons. This imbalance leads to the measurable difference in the tunneling current carried by majority- and minority-spin electrons.

The observation of spin-dependent tunneling led to the idea of a magnetic tunnel junction (MTJ) - a device that consists 
of two ferromagnetic metal layers separated by a thin insulating barrier (Figure 1a).$^{8}$ In the MTJ, the tunneling current depends on the relative orientation of the magnetizations of the two ferromagnetic layers, which can be changed by an applied magnetic field. ${ }^{9} 10$ This phenomenon is known as tunneling magnetoresistance (TMR). ${ }^{11}$ The figure of merit is the relative change in resistance of a MTJ between parallel and antiparallel magnetization orientation, known as the TMR ratio.

Since the first observation of large and reproducible TMR at room temperature, ${ }^{9}$ there has been an enormous increase of research in the field of MTJs due to their potential application in spin-electronic devices such as magnetic field sensors and magnetic random access memories (MRAMs). Significant efforts have been devoted to enhance TMR and reduce MTJ resistance by improving properties of the ferromagnetic electrode materials and the amorphous $\mathrm{Al}_{2} \mathrm{O}_{3}$ tunnel barrier. As a result, large TMR ratios up to $70 \%$ have been obtained, ${ }^{12}$ approaching the limit corresponding to the intrinsic spin polarization of $40-50 \%$ for $3 \mathrm{~d}$ ferromagnetic electrodes interfaced with amorphous $\mathrm{Al}_{2} \mathrm{O}_{3}$ barriers. ${ }^{13}$

In parallel with this endeavor, ab initio calculations have predicted very high TMR values for crystalline $\mathrm{Fe} / \mathrm{MgO} /$ $\mathrm{Fe}(001)$ tunnel junctions. ${ }^{14,15}$ This behavior is the consequence of symmetry filtering, which allows only an electronic state of the so-called $\Delta_{1}$ symmetry to tunnel efficiently from the $\mathrm{Fe}(001)$ electrode across the $\mathrm{MgO}$ barrier, while other symmetry states are filtered out. Due to the fact that the $\Delta_{1}$ symmetry state is present exclusively in the majority-spin channel at the Fermi energy, the symmetry filtering offers a virtually infinite TMR ratio for $\mathrm{Fe} / \mathrm{MgO} / \mathrm{Fe}(001)$ MTJs. Experimentally, Parkin et al. ${ }^{16}$ and Yuasa et al. ${ }^{17}$ reported TMR values in excess of $200 \%$ at room temperature in MgO-based MTJs, essentially confirming the theoretical predictions. These achievements have stimulated the race toward record TMR ratios in conjunction with low resistance-area product values that are required for application
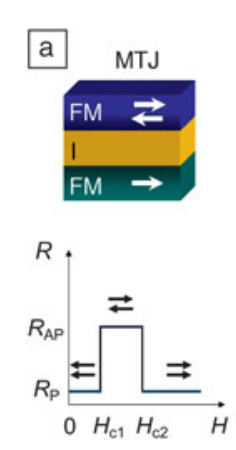
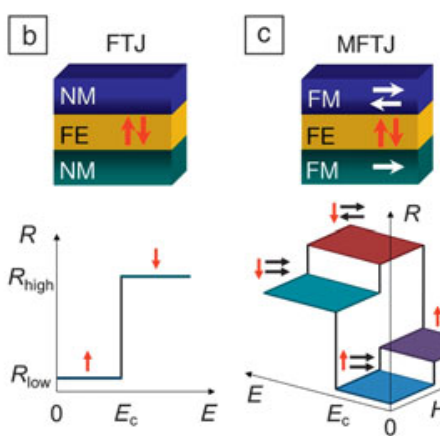

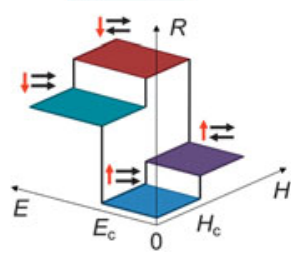

Figure 1. Schematic view of the different types of tunnel junctions: (a) magnetic tunnel junction (MTJ), (b) ferroelectric tunnel junction (FTJ), and (c) multiferroic tunnel junction (MFTJ). Ferromagnetic (FM), ferroelectric (FE), normal metal (NM), and insulating (I) layers are indicated where appropriate. Bottom panels show the resistance response of these junctions to magnetic $(H)$ and electric $(E)$ fields. Horizontal and vertical arrows indicate orientations of magnetization and electric polarization, respectively. of MTJs as sensors and MRAMs. Large TMR ratios have been reported at room temperature for $\mathrm{Co} / \mathrm{MgO} / \mathrm{Co}(410 \%)^{18}$ and $\mathrm{CoFeB} / \mathrm{MgO} / \mathrm{CoFeB}(604 \%)^{19}$ MTJs.

\section{Ferroelectric tunnel junctions}

Functional properties of tunnel junctions can be enhanced by employing a ferroelectric material as the barrier layer. Such a tunnel junction is known as a ferroelectric tunnel junction $(\text { FTJ })^{20}$ and is schematically depicted in Figure 1b. Ferroelectric materials are characterized by spontaneous electric polarization that can be switched between (at least) two stable orientations by applying an external electric field. Polarization reversal in a FTJ leads to a change in resistance of the junction, a phenomenon known as the tunneling electroresistance (TER) effect. Thus, the resistive switching of a FTJ is intimately linked to the orientation of ferroelectric polarization and hence is distinguished from other types of resistive switching known for oxide materials. ${ }^{21}$ Contrary to ferroelectric capacitors, where leakage currents are detrimental to the device performance, the conductance of a FTJ is the functional characteristic of the device.

Esaki originally proposed the concept of a polar switch involving a switchable thin-film ferroelectric material in $1971 .{ }^{22}$ However, at the time, there were no experimental techniques and capabilities to fabricate thin-film ferroelectrics to serve as a tunneling barrier. Moreover, it was believed that the critical thickness for ferroelectricity in thin films was much larger than the thickness necessary for tunneling to take place. The discovery of ferroelectricity in nanometer-thick films $\mathrm{s}^{23-25}$ opened up exciting prospects for FTJs.

The origin of the TER effect is illustrated in Figure 2. Polarization affects the interface transmission function by changing (a) the electrostatic potential across the junction, (b) interface bonding strength, and/or (c) strain associated with the piezoelectric response. ${ }^{20}$

The electrostatic effect results from incomplete screening of the polarization charges at the interface of FTJs. ${ }^{26}$ This creates

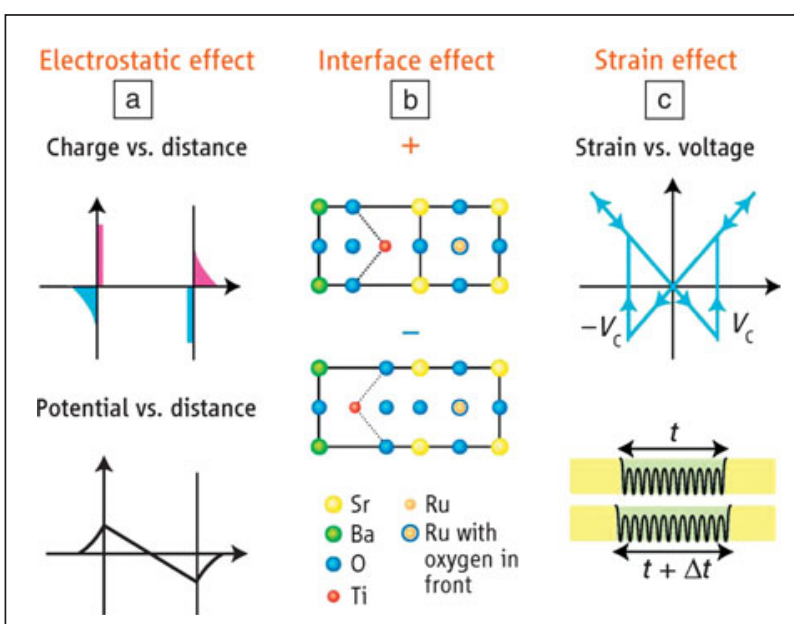

Figure 2. Mechanisms affecting tunneling conductance of ferroelectric tunnel junctions: (a) electrostatic potential at the interface, (b) interface bonding, and (c) strain. ${ }^{20}$ 
finite size charge depletion regions at the interfaces and hence an asymmetric potential profile in FTJs with different electrodes. The interface bonding effect on TER follows from atomistic calculations. ${ }^{27}$ The presence of interfaces imposes restrictions on ferroelectric displacements since the atoms at the boundary of the ferroelectric are bonded to the electrodes. The piezoelectric effect is important because all ferroelectrics are piezoelectric. Distortions along the axis of the junction, caused by applied bias, change the barrier thickness and hence affect the tunneling conductance. ${ }^{28}$ In addition, atomic displacements influence the decay rate in the barrier and consequently the transmission through it. ${ }^{27,29,30}$ We note that in FTJs with magnetic electrodes, in addition to the three mechanisms mentioned previously, an important contribution to the TER may arise from the interface magnetoelectric effect. ${ }^{31}$

Since many oxides exhibit resistive switching behavior, ${ }^{21}$ the key problem in experimentally demonstrating the polarizationcontrolled electroresistance effect is to simultaneously measure the polarization and the conductivity. Unfortunately, these measurements are often affected by defects, such as oxygen vacancies, dislocations, and grain boundaries. As a result, reliable testing of the correlation between polarization orientation and tunneling conductance is challenging. This point has been emphasized by Kohlstedt and co-workers, ${ }^{32,33}$ who showed that $I-V$ curves alone are not sufficient for the identification of the underlying resistive switching mechanism, as they could be affected by the formation of local conductive channels across a ferroelectric film.

Scanning probe microscopy (SPM) techniques allow the localization of an electric field within nanometer scale regions with simultaneous probing of polarization by piezoresponse force microscopy (PFM) ${ }^{34}$ and tunneling current by conducting atomic force microscopy (C-AFM). SPM measurements can be done in two basic geometries: with the conductive tip used as a top electrode in contact with the surface of the ferroelectric layer (Figure 3a) or with the tip contacting a deposited top electrode (Figure 3b).

One of the first studies of the polarization effect on conductivity by means of SPM involved combined detection of conduction changes in a domain-patterned $\mathrm{Pb}(\mathrm{Zr}, \mathrm{Ti}) \mathrm{O}_{3}(\mathrm{PZT})$ film. ${ }^{35}$ The large film thickness in these studies $(69 \mathrm{~nm})$ prohibited direct tunneling through the ferroelectric layer, and the observed conduction modulation was explained by band bending at the metal-ferroelectric interface due to the charge injection into the PZT film. A prototype FTJ has been demonstrated only very recently when three experimental groups independently reported experimental observations of the TER effect associated with the switching of ferroelectric polarization of $\mathrm{BaTiO}_{3}, \mathrm{PbTiO}_{3}$, and PZT films. ${ }^{36-39}$

Figure 4 illustrates the correlation between the ferroelectric polarization orientations of $\mathrm{BaTiO}_{3}$ films of different thicknesses, as is indicated by a different PFM contrast in Figure $4 \mathrm{a}-\mathrm{c}$, and the tunneling conductance across these films measured by C-AFM (Figure $4 \mathrm{~d}-\mathrm{f}$ ). ${ }^{36}$ The resistance grows exponentially with the film thickness, indicating a tunneling

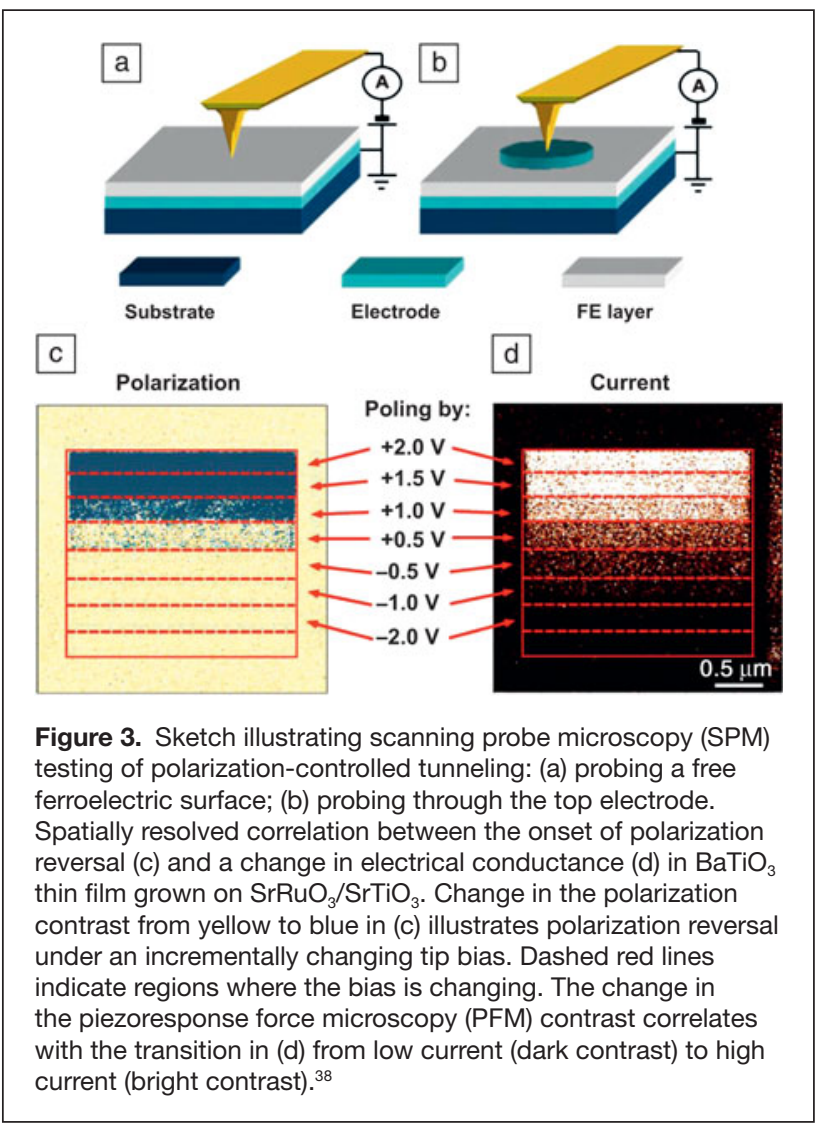

transport regime (Figure $4 \mathrm{j}$ ). TER also increased exponentially and reaches very large values up to $75,000 \%$ (Figure $4 \mathrm{k}$ ), consistent with theoretical predictions. ${ }^{26}$ Clear evidence of the link between ferroelectricity and transport is also seen from the space/bias correlation between changes in the polarization orientation and conductance (Figure 3 bottom panel). ${ }^{38}$ These experimental results prove the concept of FTJ and show the capability of thin-film ferroelectrics to serve as a nanoscale material that can act as a switch to store binary information.

Probing the TER effect through the top electrode (Figure 3b) emulates real device geometry, and for this reason is of more practical importance. Polarization stability of ultrathin ferroelectric barriers in this case becomes a more serious issue. It has been shown ${ }^{40}$ that polarization screening by metal oxide electrodes is less effective than by elemental metals, leading to progressive loss of polarization retention and may lead to a relaxation of TER. An additional complication stems from increased leakage currents due to local defects in FTJs with a relatively large electrode area. Recently, successful demonstration of room-temperature resistive switching in junctions with an $\mathrm{ON} / \mathrm{OFF}$ resistance ratio of $>10^{3}$ and lateral dimensions in the range of $0.1 \mu \mathrm{m}^{2}$ have been reported..$^{41}$

\section{Multiferroic tunnel junctions}

Multiferroic materials that are characterized by two or more ferroic orders (such as ferroelectric, ferromagnetic, ferroelastic, or ferrotoroidic) ${ }^{42}$ have recently attracted significant 


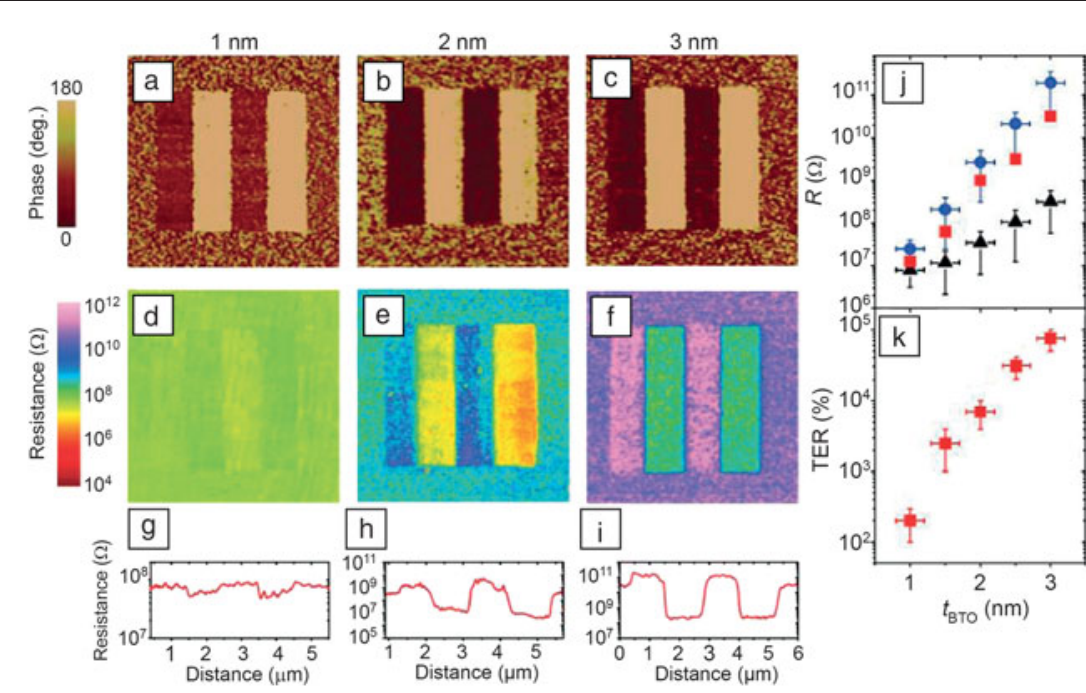

Figure 4. Observation of the giant tunneling electroresistance (TER) effect in ultrathin strained $\mathrm{BaTiO}_{3}$ films. Piezoresponse force microscopy (PFM) phase image (a-c) and conducting atomic force microscopy resistance mapping $(\mathrm{d}-\mathrm{f})$ of four written ferroelectric stripes $\left(1 \times 4 \mu \mathrm{m}^{2}\right)$ for $\mathrm{BaTiO}_{3}$ films with a thickness of 1,2 , and $3 \mathrm{~nm}$. (g-i) Corresponding resistance profiles of the poled area. (j) Thickness dependence of resistance $(R)$ of unpoled (red squares), and positively (black triangles) and negatively (blue circles) poled regions. An exponential increase in $R$ and TER $(\mathrm{k})$ with $\mathrm{BaTiO}_{3}$ thickness is seen, as expected for direct tunneling. ${ }^{36}$ interest. ${ }^{43-46}$ Among these materials, special attention has been devoted to artificial multiferroics that combine ferroelectric and ferromagnetic constituents in two-phase heterostructures..$^{47,48}$ Due to the interplay among magnetic, electric, and transport properties, these multiferroic heterostructures reveal new physics and could be used to design novel functional devices.

Multiferroic tunnel junctions (MFTJs) exploit the capability to control electron and spin tunneling via ferromagnetic and ferroelectric polarizations of the MFTJ constituents..$^{20} \mathrm{~A}$ MFTJ is a particular type of MTJ where a ferroelectric thin film serves as a tunneling barrier (Figure 1c). Equivalently, MFTJ may be considered a particular type of FTJ that has ferromagnetic metal electrodes. The key property of a MFTJ is the co-existence of the TMR and TER effects, as was predicted by Zhuravlev et al. ${ }^{49,50}$ Therefore, MFTJ represents a fourstate resistance device where resistance can be switched both by electric and magnetic fields (see the bottom panel in Figure 1c).

Density functional calculations reveal the key functional properties of MFTJs, predicting the co-existence of TMR and TER effects. ${ }^{51} \mathrm{~A}$ model MFTJ consists of perovskite oxide films: a ferroelectric $\mathrm{BaTiO}_{3}$ sandwiched between two ferromagnetic $\mathrm{SrRuO}_{3}$ electrodes (Figure 5a). The TMR effect is the consequence of wave-function symmetry conservation across the epitaxial $\mathrm{SrRuO}_{3} / \mathrm{BaTiO}_{3}$ interfaces. Majority-spin states decay inside the barrier according to the $\Delta_{1}$ symmetry, whereas the minority-spin states decay according to the $\Delta_{5}$ symmetry, yielding a perfect correspondence between symmetry and spin (Figure 5b-c). Thus, in the parallel magnetic configuration, both spin channels contribute to the conductance. In the antiparallel magnetic configuration, the conductance is strongly suppressed due to the symmetry mismatch. This yields a sizable TMR.

The TER effect originates from the asymmetric interface termination sequence $\left(\mathrm{RuO}_{2} / \mathrm{BaO}\right.$ at one interface versus $\mathrm{TiO}_{2} / \mathrm{SrO}$ at the other) that creates a different polarization profile when the ferroelectric polarization is switched..$^{51}$ This gives rise to a change in the magnitude of the bandgap in $\mathrm{BaTiO}_{3}$ and therefore modifies the tunneling decay rate (Figure 5c), resulting in TER. There is also an effect of ferroelectric polarization on tunneling spin polarization and TMR. The $\Delta_{1}$ and $\Delta_{5}$ bands carry the majority and minority states from the $\mathrm{SrRuO}_{3}$ electrodes. Since these states are affected differently when the ferroelectric polarization is switched, and the tunneling transport depends exponentially on the decay rate, this leads to a significant change in TMR. In addition, the magnetoelectric effect at the $\mathrm{SrRuO}_{3} / \mathrm{BaTiO}_{3}$ interface changes the exchange splitting of the $\mathrm{SrRuO}_{3}$ spin-bands, ${ }^{52}$ affecting the spin polarization and TMR.

Experimentally, the impact of ferroelectric polarization of the barrier on spin-dependent tunneling has been demonstrated 
by Garcia et al. ${ }^{53}$ They fabricated MFTJs composed of an $\mathrm{La}_{2 / 3} \mathrm{Sr}_{1 / 3} \mathrm{MnO}_{3}$ (LSMO) electrode, a $\mathrm{BaTiO}_{3}$ ferroelectric tunnel barrier (1 to $3 \mathrm{~nm}$ ), and a Fe or Co counter-electrode. At $4 \mathrm{~K}$, a large negative TMR (Figure 6a) was found, reflecting a negative spin polarization for the $\mathrm{Fe} / \mathrm{BaTiO}_{3}$ interface. By applying short voltage pulses of $\pm 1 \mathrm{~V}$, they observed reversible changes of the tunnel resistance linked to the variation of the barrier height with the ferroelectric polarization direction (Figure 6b), with a TER of about 30\%. More interestingly, the amplitude of the TMR was also found to strongly depend on the direction of the ferroelectric polarization. As can be seen for a typical junction in Figure 6c, the TMR measured at an applied bias voltage of $-50 \mathrm{mV}$ varies from a high value $(-17 \%)$ to a low value $(-3 \%)$ when the electrical polarization points toward Fe or LSMO, respectively. Considering its half metallic nature, LSMO has poor sensitivity to the ferroelectric polarization direction. This is supported by the fact that such modulation of the TMR effect by ferroelectricity in LSMO/(La,Bi) $\mathrm{MnO}_{3} / \mathrm{Au}^{54}$ tunnel junctions was not observed. The observed change in TMR is consistent with the predicted change of the spin polarization at the $\mathrm{Fe} / \mathrm{BaTiO}_{3}$ interface ${ }^{55}$ and the induced magnetic moment on the interface Ti atoms. ${ }^{55,56}$ These results reveal that the spin polarization of tunneling electrons can be electrically tuned in MFTJs through reversal of the ferroelectric polarization of the barrier. Experimental evidence that the transport spin polarization can be controlled by the switchable ferroelectric polarization was also demonstrated for MFTJs based on LSMO electrodes with ferroelectric $\mathrm{Ba}_{0.95} \mathrm{Sr}_{0.05} \mathrm{TiO}_{3}{ }^{57}$ and $\mathrm{BiFeO}_{3}{ }^{58}$ tunnel barriers.

\section{Outlook}

The realization of ferroelectric and multiferroic tunnel junctions promises exciting technological applications. In recent years, there has been a surge in research activities aimed at investigating the next generation of memory/logic devices that would overcome scaling limitations of conventional semiconductor technology based on charge storage ${ }^{59}$ One of the key challenges is to develop a switch involving a significant bi-stable effect that can be controlled by an external stimulus and is robust enough to be harnessed as manufacturable technology. The giant change of resistance in FTJs associated with polarization switching promises a new approach to the electrical switching of resistance that can be used in non-charge-based memory and logic devices. ${ }^{60}$ In addition, FTJs employ non-destructive read operation, ${ }^{36}$ opening the door for faster and energy-efficient random access memories. Furthermore, conceptually new multilevel memory and logic devices are possible with MFTJs.

Although the potential impact of these technologies is tremendous, a number of scientific issues remain to be resolved to produce commercially viable devices. Fundamental issues that need to be addressed for further advances in the field include the stability of ferroelectric ordering in ultrathin films and its relationship to electrical and mechanical boundary conditions. Due to the electronic and transport properties of FTJs and MFTJs being inherently related to the ferroelectric polarization, the fundamental mechanisms of ferroelectric switching and the effect of structural and interfacial defects on electronic transport properties are also of critical importance. As a result of the pronounced scaling effect in ferroelectrics, transition to heterostructures with nanoscale lateral dimensions will likely entail significant changes in their properties, so that the issues of ferroelectric and transport behavior in FTJs and MFTJs may need to be readdressed at the appropriate length scale.

The magnetic properties of ferromagnetic materials employed in MFTJs are critical for the operation of these
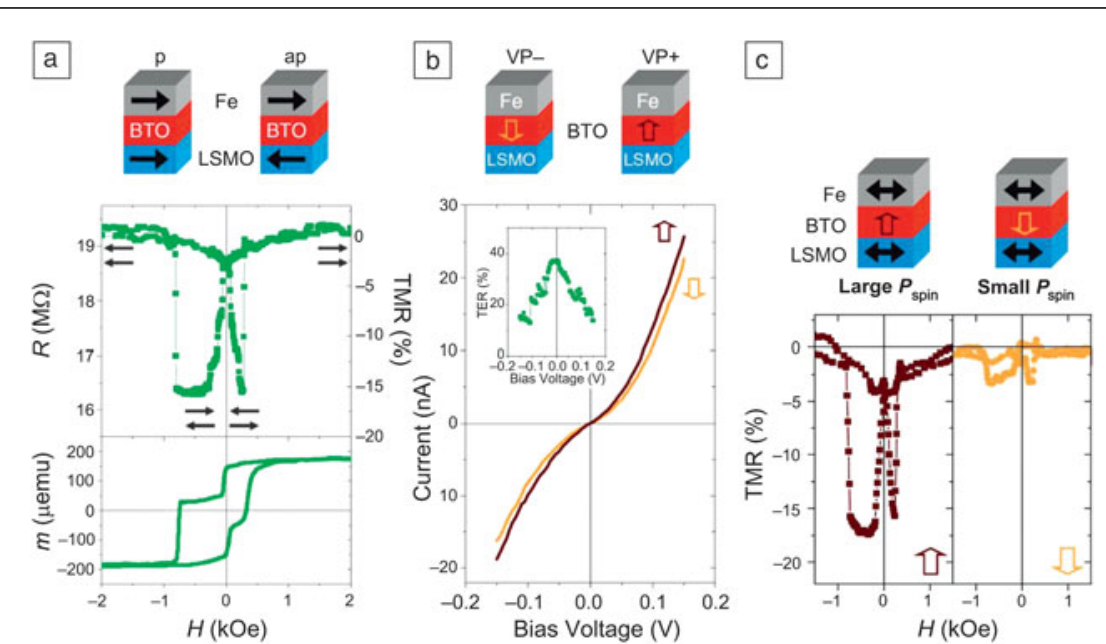

Figure 6. Magnetoresistive and electroresistive properties of $\mathrm{Fe} / \mathrm{BaTiO}_{3}(1 \mathrm{~nm}) / \mathrm{LSMO}$ multiferroic tunnel junctions. (a) Resistance (top) and magnetic moment (bottom) as a function of the magnetic field. (b) $I-V$ characteristics of the junction recorded at $4 \mathrm{~K}$ after poling the ferroelectric $\mathrm{BaTiO}_{3}$ barrier up and down. (c) Tunneling magnetoresistance (TMR) curves after poling the ferroelectric tunnel barrier up and down. A clear modulation of the TMR with the ferroelectric polarization orientation is seen. ${ }^{53}$ devices. The essential role is played by interfaces where a magnetically dead layer may occur due to either non-stoichiometry or defects formed at the interfaces, suppressing the transport spin-polarization and TMR. Magnetization switching behavior of the magnetic thin films in a MFTJ is also important and interesting due to a possible effect of the ferroelectric polarization on interface magnetic anisotropy and coercivity of the film. The interrelationship between ferroelectricity of the barrier layer and ferromagnetism of the electrodes through an interface magnetoelectric effect may also control other functional properties in MFTJs, such as TMR and TER, and needs to be investigated in depth. Although meeting these challenges is not easy, the prediction and recent demonstration of giant resistive switching effects and the control of tunneling spin polarization are a strong testament to the fact that the field of ferroelectric and multiferroic tunnel junctions has an exciting future. 


\section{References}

1. J. Frenkel, Phys. Rev. 36, 1604 (1930).

2. B.D. Josephson, Rev. Mod. Phys. 46, 251 (1974).

3. G. Binnig, H. Rohrer, Rev. Mod. Phys. 59, 615 (1987).

4. R.H. Fowler, L. Nordheim, Proc. R. Soc. London 119, 173 (1928).

5. C. Chappert, A. Fert, F.N. Van Dau, Nat. Mater. 6, 813 (2007).

6. E.Y. Tsymbal, I. Žutić, Eds. Handbook of Spin Transport and Magnetism, (Taylor \& Francis, NY, 2011).

7. P.M. Tedrow, R. Meservey, Phys. Rev. Lett. 26, 192 (1971).

8. M. Jullière, Phys. Lett. A 54, 225 (1975).

9. J.S. Moodera, L.R. Kinder, T.M. Wong, R. Meservey, Phys. Rev. Lett. 74, 3273 (1995).

10. T. Miyazaki, N.J. Tezuka, J. Magn. Mag. Mater. 139, L231 (1995).

11. E.Y. Tsymbal, O.N. Mryasov, P.R. LeClair, J. Phys.: Condens. Matter 15 R109 (2003); E.Y. Tsymbal, K.D. Belashchenko, J. Velev, S.S. Jaswal, M. van Schilfgaarde, I.I. Oleynik, D.A. Stewart, Prog. Mater. Science. 52, 401 (2007). 12. D. Wang, C. Nordman, J. Daughton, Z. Qian, J. Fink, IEEE Trans. Magn. 40, 2269 (2004)

13. D.J. Monsma, S.S.S. Parkin, Appl. Phys. Lett. 77, 720 (2000)

14. W.H. Butler, X.-G. Zhang, T.C. Schulthess, J.M. MacLaren, Phys. Rev. B 63, 054416 (2001)

15. J. Mathon, A. Umerski, Phys. Rev. B 63, R220403 (2001)

16. S.S.P. Parkin, C. Kaiser, A. Panchula, P.M. Rice, B. Hughes, Nat. Mater. 3 , $862(2004)$

17. S. Yuasa, T. Nagahama, A. Fukushima, Y. Suzuki, K. Ando, Nat. Mater. 3 , 868 (2004)

18. S. Yuasa, A. Fukushima, H. Kubota, Y. Suzuki, K. Ando, Appl. Phys. Lett. 89, 042505 (2006)

19. S. Ikeda, J. Hayakawa, Y. Ashizawa, Y.M. Lee, K. Miura, H. Hasegawa, M. Tsunoda, F. Matsukura, H. Ohno, Appl. Phys. Lett. 93, 082508 (2008).

20. E.Y. Tsymbal, H. Kohlstedt, Science 313, 181 (2006).

21. R. Waser, M. Aono, Nat. Mater. 6, 833 (2007).

22. L. Esaki, R.B. Laibowitz, P.J. Stiles, IBM Tech. Discl. Bull. 13, 2161 (1971). 23. D.D. Fong, G.B. Stephenson, S.K. Streiffer, J.A. Eastman, O. Auciello, P.H. Fuoss, C. Thompson, Science 304, 1650 (2004).

24. C. Lichtensteiger, J.-M. Triscone, J. Junquera, P. Ghosez, Phys. Rev. Lett. 94, 047603 (2005)

25. D.A. Tenne, A. Bruchhausen, N.D. Lanzillotti-Kimura, A. Fainstein, R.S. Katiyar, A. Cantarero, A. Soukiassian, V. Vaithyanathan, J.H. Haeni, W. Tian, D.G. Schlom, K.J. Choi, D.M. Kim, C.B. Eom, H.P. Sun, X.Q. Pan, Y.L. Li, L.Q. Chen, Q.X. Jia, S.M. Nakhmanson, K.M. Rabe, X.X. Xi, Science 313, 1614 (2006)

26. M.Y. Zhuravlev, R.F. Sabirianov, S.S. Jaswal, E.Y. Tsymbal, Phys. Rev. Lett. 94, 246802 (2005); Phys. Rev. Lett. 102, 169901 (2009)

27. J.P. Velev, C.-G. Duan, K.D. Belashchenko, S.S. Jaswal, E.Y. Tsymbal, Phys. Rev. Lett. 98, 137201 (2007).

28. H. Kohlstedt, N.A. Pertsev, J. Rodríguez Contreras, R. Waser, Phys. Rev. B 72, 125341 (2005).

29. N.F. Hinsche, M. Fechner, P. Bose, S. Ostanin, J. Henk, I. Mertig, P. Zahn, Phys. Rev. B 82, 214110 (2010).

30. D. Wortmann, S. Blügel, Phys. Rev. B 83, 155114 (2011).

31. J.D. Burton, E.Y. Tsymbal, Phys. Rev. B 80, 174406 (2009); Phys. Rev. Lett. 106, 157203 (2011).

32. H. Kohlstedt, A. Petraru, K. Szot, A. Ruediger, P. Meuffels, H. Haselier, R. Waser, V. Nagarajan, Appl. Phys. Lett. 92, 062907 (2008).

33. J. Rodríguez Contreras, H. Kohlstedt, U. Poppe, R. Waser, C. Buchal, N.A. Pertsev, Appl. Phys. Lett. 83, 4595 (2003).
34. A. Gruverman, 0. Auciello, H. Tokumoto, Annu. Rev. Mater. Sci. 28, 101 (1998)

35. C. Yoshida, A. Yoshida, H. Tamura, Appl. Phys. Lett. 75, 1449 (1999).

36. V. Garcia, S. Fusil, K. Bouzehouane, S. Enouz-Vedrenne, N.D. Mathur, A. Barthélémy, M. Bibes, Nature 460, 81 (2009).

37. P. Maksymovych, S. Jesse, P. Yu, R. Ramesh, A.P. Baddorf, S.V. Kalinin Science 324, 1421 (2009).

38. A. Gruverman, D. Wu, H. Lu, Y. Wang, H.W. Jang, C.M. Folkman, M.Y. Zhuravlev, D. Felker, M. Rzchowski, C.-B. Eom, E.Y. Tsymbal, Nano Lett. 9, 3539 (2009). 39. A. Crassous, V. Garcia, K. Bouzehouane, S. Fusil, A.H.G. Vlooswijk, G. Rispens, B. Noheda, M. Bibes, A. Barthélémy, Appl. Phys. Lett. 96, 042901 (2010).

40. V. Nagarajan, J. Junquera, J.Q. He, C.L. Jia, R. Waser, K. Lee, Y.K. Kim, S. Baik, T. Zhao, R. Ramesh, P. Ghosez, K.M. Rabe, J. Appl. Phys. 100, 051609 (2006).

41. D. Pantel, S. Goetze, D. Hesse, M. Alexe, ACS Nano 5, 6032 (2011) A. Chanthbouala, A. Crassous, V. Garcia, K. Bouzehouane, S. Fusil, X. Moya, J. Allibe, B. Dlubak, J. Grollier, S. Xavier, C. Deranlot, A. Moshar, R. Proksch, N.D. Mathur, M. Bibes, A. Barthélémy, Nature Nanotech. doi: 10.1038/nnano. 2011.213.

42. H. Schmid, Ferroelectrics 161, 1 (1994).

43. M. Fiebig, J. Phys. D 38, R123 (2005)

44. W. Eerenstein, N.D. Mathur, J.F. Scott, Nature 442, 759 (2006).

45. R. Ramesh, N.A. Spaldin, Nat. Mater. 6, 21 (2007)

46. M. Bibes, A. Barthélémy, IEEE Trans. Electron Devices 54, 1003 (2007).

47. K.F. Wang, J.-M. Liu, Z.F. Ren, Adv. Phys. 58, 321 (2009)

48. J.P. Velev, S.S. Jaswal, E.Y. Tsymbal, Philos. Trans. R. Soc. London, Ser. A 369, 3069 (2011).

49. M.Y. Zhuravlev, S.S. Jaswal, E.Y. Tsymbal, R.F. Sabirianov, Appl. Phys. Lett. 87, 222114 (2005)

50. M.Y. Zhuravlev, S. Maekawa, E.Y. Tsymbal, Phys. Rev. B 81, 104419 (2010). 51. J.P. Velev, C.-G. Duan, J.D. Burton, A. Smogunov, M.K. Niranjan, E. Tosatti, S.S. Jaswal, E.Y. Tsymbal, Nano Lett. 9, 427 (2009).

52. M.K. Niranjan, J.D. Burton, J.P. Velev, S.S. Jaswal, E.Y. Tsymbal, Appl. Phys. Lett. 95, 052501 (2009).

53. V. Garcia, M. Bibes, L. Bocher, S. Valencia, F. Kronast, S. Enouz-Vedrenne, A. Gloter, D. Imhoff, C. Deranlot, N.D. Mathur, S. Fusil, K. Bouzehouane, A. Barthélémy, Science 327, 1106 (2010).

54. M. Gajek, M. Bibes, S. Fusil, K. Bouzehouane, J. Fontcuberta, A. Barthélémy, A. Fert, Nat. Mater. 6, 296 (2007).

55. C.-G. Duan, S.S. Jaswal, E.Y. Tsymbal, Phys. Rev. Lett. 97, 047201 (2006). 56. S. Valencia, A. Crassous, L. Bocher, V. Garcia, X. Moya, R.O. Cherifi, C. Deranlot, K. Bouzehouane, S. Fusil, A. Zobelli, A. Gloter, N.D. Mathur, A. Gaupp, R. Abrudan, F. Radu, A. Barthélémy, M. Bibes, Nat. Mater. 10, 753 (2011).

57. Y.W. Yin, M. Raju, W.J. Hu, X.J. Weng, X.G. Li, Q. Li, J. Appl. Phys. 109 07 D915 (2011).

58. M. Hambe, A. Petraru, N.A. Pertsev, P. Munroe, V. Nagarajan, H. Kohlstedt, Adv. Funct. Mater. 20, 2436 (2010)

59. J.A. Hutchby, R. Cavin, V. Zhirnov, J.E. Brewer, G. Bourianoff, Computer 41, 28 (2008).

60. P. Zubko, J.-M. Triscone, Nature 460, 45 (2009).

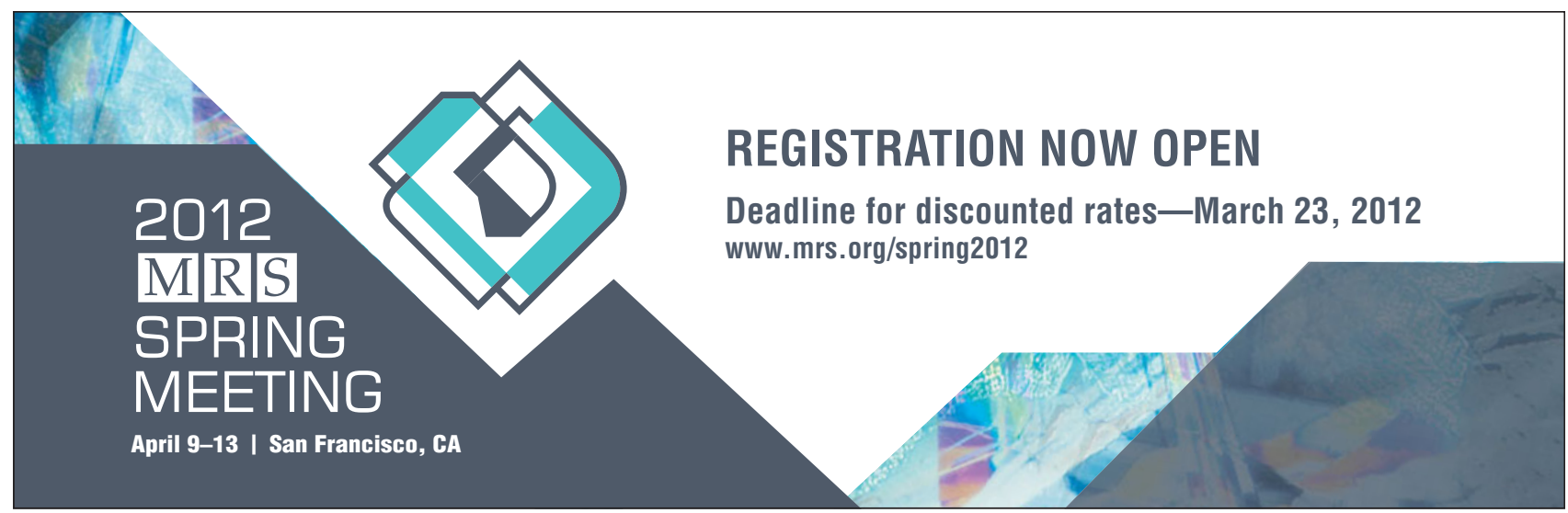

